UCRL-ID-153593

\title{
Level Densities and Radiative Strength Functions in ${ }^{170,171} \mathrm{Yb}$
}

U. Agvaanluvsan, A. Schiller, J. A. Becker, L. A. Bernstein, M. Guttormsen, G. E. Mitchell, J. Rekstad, S. Siem, A. Voinov

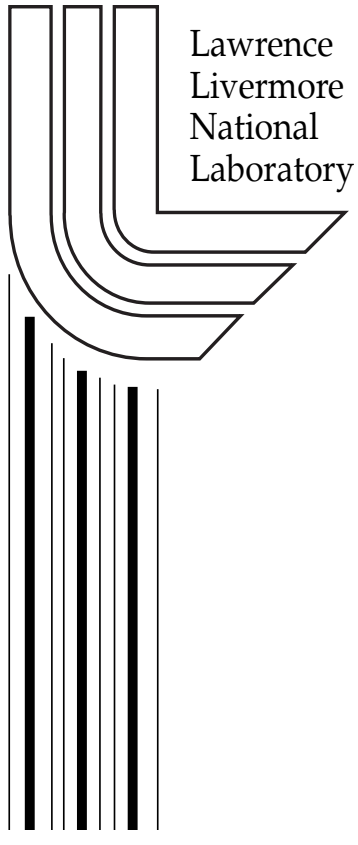

June 9, 2003 
This document was prepared as an account of work sponsored by an agency of the United States Government. Neither the United States Government nor the University of California nor any of their employees, makes any warranty, express or implied, or assumes any legal liability or responsibility for the accuracy, completeness, or usefulness of any information, apparatus, product, or process disclosed, or represents that its use would not infringe privately owned rights. Reference herein to any specific commercial product, process, or service by trade name, trademark, manufacturer, or otherwise, does not necessarily constitute or imply its endorsement, recommendation, or favoring by the United States Government or the University of California. The views and opinions of authors expressed herein do not necessarily state or reflect those of the United States Government or the University of California, and shall not be used for advertising or product endorsement purposes. 


\title{
Level densities and radiative strength functions in ${ }^{170,171} \mathbf{Y b}$
}

\author{
U. Agvaanluvsan* $^{*}$ A. Schiller ${ }^{\dagger}$, J.A. Becker ${ }^{\dagger}$, L.A. Bernstein ${ }^{\dagger}$, M. Guttormsen**, \\ G. E. Mitchell*, J. Rekstad**, S. Siem** and A. Voinov ${ }^{\ddagger}$ \\ ${ }^{*}$ North Carolina State University, Raleigh, NC 27695 \\ and Triangle Universities Nuclear Laboratory, Durham, NC 27708 \\ ${ }^{\dagger}$ L-414, Lawrence Livermore National Laboratory, 7000 East Ave., Livermore, CA 94551 \\ ${ }^{* *}$ Department of Physics, University of Oslo, $\mathrm{N}-0316$ Oslo, Norway \\ ${ }^{\ddagger}$ Frank Laboratory of Neutron Physics, Joint Institute of Nuclear Research, 141980 Dubna, Moscow region, \\ Russia
}

\begin{abstract}
Level densities and radiative strength functions in ${ }^{171} \mathrm{Yb}$ and ${ }^{170} \mathrm{Yb}$ nuclei have been measured with the ${ }^{171} \mathrm{Yb}\left({ }^{3} \mathrm{He},{ }^{3} \mathrm{He}^{\prime} \gamma\right){ }^{171} \mathrm{Yb}$ and ${ }^{171} \mathrm{Yb}\left({ }^{3} \mathrm{He}, \alpha \gamma\right){ }^{170} \mathrm{Yb}$ reactions. A simultaneous determination of the nuclear level density and the radiative strength function was made. The present data adds to and is consistent with previous results for several other rare earth nuclei. The method will be briefly reviewed and the result from the analysis will be presented. The radiative strength function for ${ }^{171} \mathrm{Yb}$ is compared to previously published work.
\end{abstract}

\section{INTRODUCTION}

Nuclear level densities and radiative strength functions are important inputs for calculations of nuclear reaction rates. Much of nuclear density data is available from counting of low-lying levels. With increasing excitation energy, the level density becomes large and individual levels are no longer resolvable [1]. Another basis of level density data comes from nuclear resonance spacings at or above the nucleon binding energy [2]. Between these two excitation energy regions, relatively little is known about nuclear level densities.

A major part of the information on radiative strength functions comes from the photoabsorption cross section measurements [3]. High energy $\gamma$ transitions with $E_{\gamma} \sim 10-15 \mathrm{MeV}$ are dominated by the giant electric dipole resonance (GEDR). Although the electric dipole transition strengths are well studied in the vicinity of the GEDR, the behavior at $E_{\gamma}<\mathrm{B}_{\mathrm{n}}$ is not well determined [4]. Experimental data on the $M 1$ strength function is much scarcer than for the $E 1$ strength function. A recent experimental technique developed by the Oslo Cyclotron Group allows one to determine level densities and radiative strength functions simultaneously $[5,6]$. The advantage of this method is that it provides data on nuclear level densities and radiative strength functions in regions where there is scarcity of data and few experimental means available. The disadvantage is that the level density and radiative strength function are coupled. The discrete states, the resonance spacing and capture data are needed for normalization. This method is commonly referred to as the Oslo method. It has been proven to work well in heavy mass nuclei and is currently being investigated in other mass regions. The present paper reports on results from a ${ }^{171} \mathrm{Yb}+{ }^{3} \mathrm{He}$ experiment. The Oslo method and the experimental set-up are briefly discussed, followed by a brief description of level densities and radiative strength functions. Results from the analysis of the present measurements are presented and discussed in the last two sections.

\section{METHOD}

The experiment was carried out at the Oslo Cyclotron Laboratory (OCL) using a $45 \mathrm{MeV}{ }^{3} \mathrm{He}$ beam. The self-supporting ${ }^{171} \mathrm{Yb}$ target had a thickness of $1.6 \mathrm{mg} \mathrm{cm}^{-2}$. The two reactions ${ }^{171} \mathrm{Yb}\left({ }^{3} \mathrm{He},{ }^{3} \mathrm{He}^{\prime} \gamma\right){ }^{171} \mathrm{Yb}$ and ${ }^{171} \mathrm{Yb}\left({ }^{3} \mathrm{He}, \alpha \gamma\right){ }^{170} \mathrm{Yb}$ were the focus of the experiment. Particle- $\gamma$ coincidences for ${ }^{170,171} \mathrm{Yb}$ were detected using the CACTUS multidetector array. The charged particles were measured with eight Si particle telescopes placed at $45^{\circ}$ 
with respect to the beam direction. Each telescope consists of a front $\mathrm{Si} \Delta E$ detector and a back $\mathrm{Si}(\mathrm{Li}) E$ detector with thicknesses 140 and $3000 \mu \mathrm{m}$, respectively. An array of 28 collimated $\mathrm{NaI} \gamma$-ray detectors with a total coverage of $\sim 15 \%$ of $4 \pi$ surrounds the target. In addition three Ge detectors were used to estimate the spin distribution and determine the selectivity of the reaction. The typical spin range is $I \sim 2-6 \hbar$.

Reaction kinematics are used to transform particle energy to nuclear excitation energy. For each excitation energy total cascade $\gamma$-ray spectra are obtained from the coincidence measurement. The $\gamma$-ray spectrum is unfolded using the responce matrix [6]. The $\gamma$-ray spectrum containing only the first $\gamma$ rays emitted from a given excitation energy is called the first-generation spectrum. For each excitation energy window, the corresponding first generation spectrum is obtained using the extraction described in ref. [7]. A matrix is formed whose entries are the probabilities $P\left(E_{x}, E_{\gamma}\right)$ that a $\gamma$-ray of energy $E_{\gamma}$ is emitted from excitation energy $E_{x}$.

The probability of $\gamma$ decay is proportional to the product of the $\gamma$-ray strength (i.e., the radiative transmission coefficient $\left.F\left(E_{\gamma}\right)\right)$ and the level density $\rho\left(E_{x}-E_{\gamma}\right)$ at the final energy $E_{x}-E_{\gamma}$

$$
P\left(E_{x}, E_{\gamma}\right) \propto F\left(E_{\gamma}\right) \rho\left(E_{x}-E_{\gamma}\right),
$$

This factorization is the generalized form of the Brink-Axel hypothesis $[8,9]$. The functions $\rho$ and $F$ are determined by an iteration procedure in the Oslo method [5]. The goal of the iteration is to determine these two functions at $N$ energies (thus $2 N$ values); the product of the two functions is known at roughly $N^{2}$ data points. The globalized fitting to the data points determines the functional form for $\rho$ and $F$ [5]. With the functional form available one determines the absolute values for $\rho$ and $F$ by comparison with other experimental data. The level density $\rho$ is determined from the nuclear ground state up to $\sim B_{n}-1 \mathrm{MeV}$ where $B_{n}$ is the neutron binding energy. The radiative transmission coefficient $F$ is obtained from $E_{\gamma} \approx 1 \mathrm{MeV}$ to around $B_{n} \mathrm{MeV}$.

\section{LEVEL DENSITY AND RADIATIVE STRENGTH FUNCTION}

The level density at low excitation energy in the final nucleus is small and experimental measurements of low-lying levels are available for most stable nuclei. Figure 1 shows the level density at low excitation energy determined from the present experimental data compared to the discrete levels listed in the Table of Isotopes [10]. The agreement is good up to $E \sim 1.5 \mathrm{MeV}$. Above this energy the two results differ because there is limited information on discrete levels at higher excitation energy. The present experiment provides new results for the average level density for $E \geq$ $1.5 \mathrm{MeV}$. Comparison at low excitation energy is used to fix the absolute value of the data at the low-energy end. The level density at $B_{n}$ determined from neutron resonance spacings is employed as a normalization point at the high-energy end of our measurement.

The $\gamma$-ray transmission coefficient $F\left(E_{\gamma}\right)$ in Eq. 1 is expressed in terms of a sum of all the $\gamma$-ray strength functions $f_{X L}$ of multipolarities $X L$

$$
F\left(E_{\gamma}\right) \propto \sum_{X L} E^{2 L+1} f_{X L}\left(E_{\gamma}\right) .
$$

The average radiative width of neutron resonances $\left\langle\Gamma_{\gamma}\right\rangle$ at the neutron binding energy is used to normalize $F\left(E_{\gamma}\right)$. One assumes that the experimental value of $\left\langle\Gamma_{\gamma}\right\rangle$ has contributions from $I \pm 1 / 2$, where $I$ is the target spin in the weighting factor that determines the normalization to $F\left(E_{\gamma}\right)$ [11].

It is assumed that the $\gamma$-ray strength is dominated by dipole transitions. The Lorentzian giant electric dipole resonance (GEDR) and the Kadmensky-Markushev-Furman (KMF) models are employed for the $E 1$ strength. According to the Brink hypothesis which assumes that properties of the GEDR built on excited states are equal to those built on the ground state, the GEDR can be expressed as

$$
f_{E 1}\left(E_{\gamma}\right)=\frac{1}{3 \pi^{2} \hbar^{2} c^{2}} \frac{\sigma_{E 1} E_{\gamma} \Gamma_{E 1}^{2}}{\left(E_{\gamma}^{2}-E_{E 1}^{2}\right)^{2}+E_{\gamma}^{2} \Gamma_{E 1}^{2}},
$$

where $\sigma_{E 1}, \Gamma_{E 1}$, and $E_{E 1}$ are the cross section, width, and the centroid of the GEDR determined by photoabsorption. In the KMF model [12], the Lorentzian GEDR is modified in order to reproduce the non-zero tail of the GEDR for $E_{\gamma} \rightarrow 0$ by means of a temperature dependent width of the GEDR. The $E 1$ strength in the KMF model is given by

$$
f_{E 1}\left(E_{\gamma}\right)=\frac{1}{3 \pi^{2} \hbar^{2} c^{2}} \frac{0.7 \sigma_{E 1} \Gamma_{E 1}^{2}\left(E_{\gamma}^{2}+4 \pi^{2} T^{2}\right)}{E_{E 1}\left(E_{\gamma}^{2}-E_{E 1}^{2}\right)^{2}},
$$




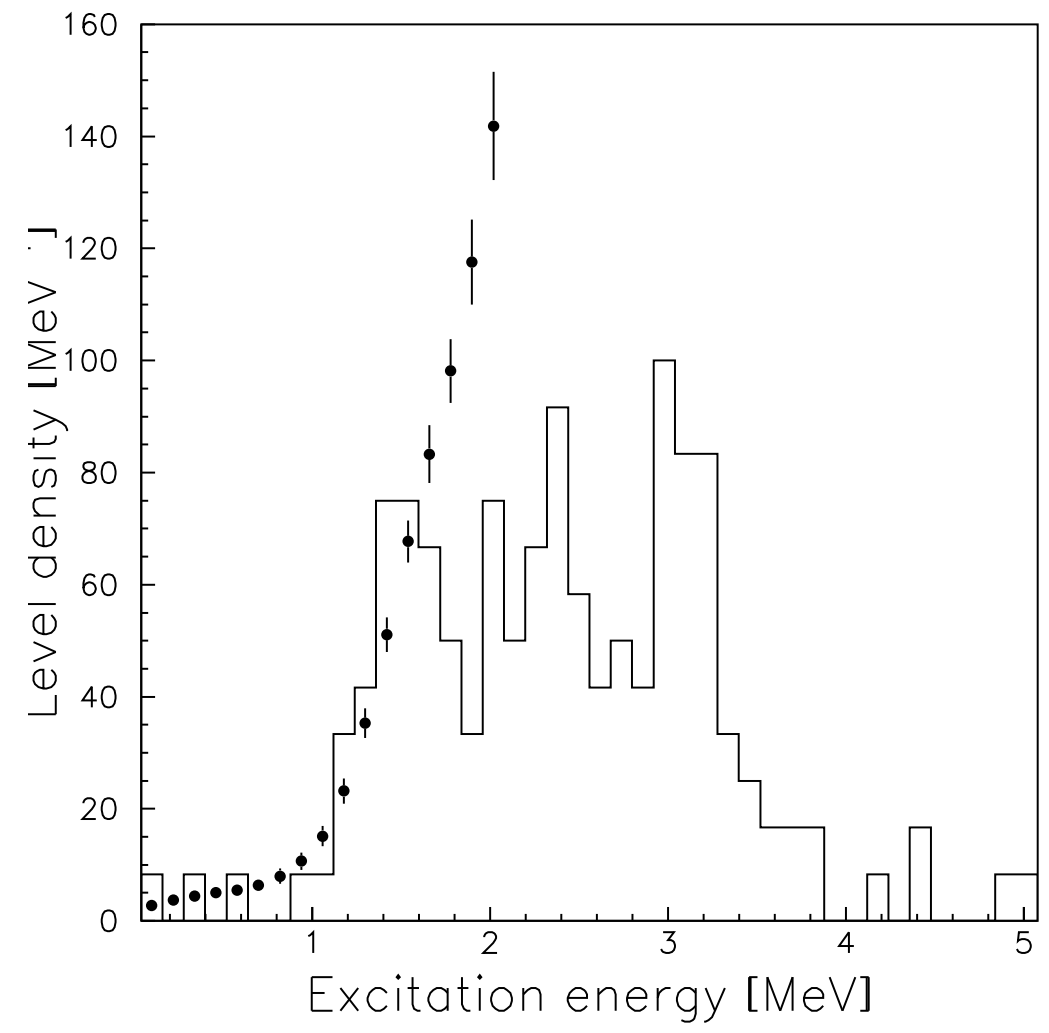

FIGURE 1. The level density of ${ }^{170} \mathrm{Yb}$ (data points) compared to that obtained from the Table of Isotopes (histograms).

where $T=\sqrt{U / a}$ is the nuclear temperature, $U$ is the effective excitation energy, and $a$ is the Fermi gas level density parameter. The width of the GEDR is a sum of energy and temperature dependent parts

$$
\Gamma_{E 1}\left(E_{\gamma}, T\right)=\frac{\Gamma_{E 1}}{E_{E 1}^{2}}\left(E_{\gamma}^{2}+4 \pi^{2} T^{2}\right) .
$$

The giant dipole resonance is split into two parts for deformed nuclei. Therefore, a sum of two strength functions is used.

Two different descriptions of $M 1$ strength are considered in comparison with data. The first is Weisskopf's adjusted single particle model, where the $M 1$ strength $f_{M 1}$ is independent of $\gamma$-ray energy and its value is determined from the ratio $f_{M 1} / f_{E 1}$ at $E_{\gamma} \approx 7 \mathrm{MeV}$ [13]. The second model for $f_{M 1}$ is the Lorentzian giant magnetic dipole resonance (GMDR) given by

$$
f_{M 1}\left(E_{\gamma}\right)=\frac{1}{3 \pi^{2} \hbar^{2} c^{2}} \frac{\sigma_{M 1} E_{\gamma} \Gamma_{M 1}^{2}}{\left(E_{\gamma}^{2}-E_{M 1}^{2}\right)^{2}+E_{\gamma}^{2} \Gamma_{M 1}^{2}} .
$$

For several rare-earth nuclei, an anomalous resonance structure is observed in the radiative strength function. These are similar to the giant resonances but with smaller width [11,14]. This structure is often refered to as a pygmy resonance. The electromagnetic character of this structure is currently under investigation. In order to reproduce experimental results where this structure is evident, another Lorentzian centered at $E_{\mathrm{py}}$ with width $\Gamma_{\mathrm{py}}$ and cross section $\sigma_{\mathrm{py}}$ is used in addition to the GEDR and GMDR described above. 


\section{RESULTS AND DISCUSSION}

The level density and strength function for ${ }^{170} \mathrm{Yb}$ reported in this paper are new results. For ${ }^{171} \mathrm{Yb}$, the level density and radiative strength function were obtained previously from a ${ }^{172} \mathrm{Yb}\left({ }^{3} \mathrm{He}, \alpha \gamma\right){ }^{171} \mathrm{Yb}$ experiment [11]. Figure 2 shows the level densities of ${ }^{170} \mathrm{Yb}$ and ${ }^{171} \mathrm{Yb}$. Data points are drawn as full circles while level densities at the neutron binding energy are represented by empty circles. Results for both nuclei are obtained from the ground state to $\sim B_{n}-1 \mathrm{MeV}$. The solid line is an interpolation between the experimental result and $\rho$ evaluated at $B_{n}$ using a back-shifted Fermi gas approximation for the level density

$$
\rho(E)=\eta \frac{\exp (2 \sqrt{a U})}{12 \sqrt{2} a^{1 / 4} U^{5 / 4} \sigma_{I}} .
$$

The back-shifted excitation energy $U=E-C-\Delta$, where $C=-6.6 A^{-0.32} \mathrm{MeV}, A$ is the mass number, and $\Delta$ is the pairing parameter. The $\Delta$ parameter is estimated to be $\Delta=\Delta_{n}+\Delta_{p}=1.405 \mathrm{MeV}$ for ${ }^{170} \mathrm{Yb}$ and $\Delta_{p}=0.319$ $\mathrm{MeV}$ for ${ }^{171} \mathrm{Yb}$, following the prescription by Dobaczewski et al. [15]. The level density for ${ }^{170} \mathrm{Yb}$ is about an order of magnitude smaller than the level density for ${ }^{171} \mathrm{Yb}$. This is consistent with a larger shift for the level density for this even-even nucleus. Plateaus in the level densities around $E=\Delta$ may be related to nucleon pair breaking. This signature of pair breaking is commonly observed in other rare-earth nuclei [16] as well as in lighter nuclei [17] studied by the Oslo method. The theoretical interpretation that relates the pair breaking with thermodynamical properties is given in a recent review [18].

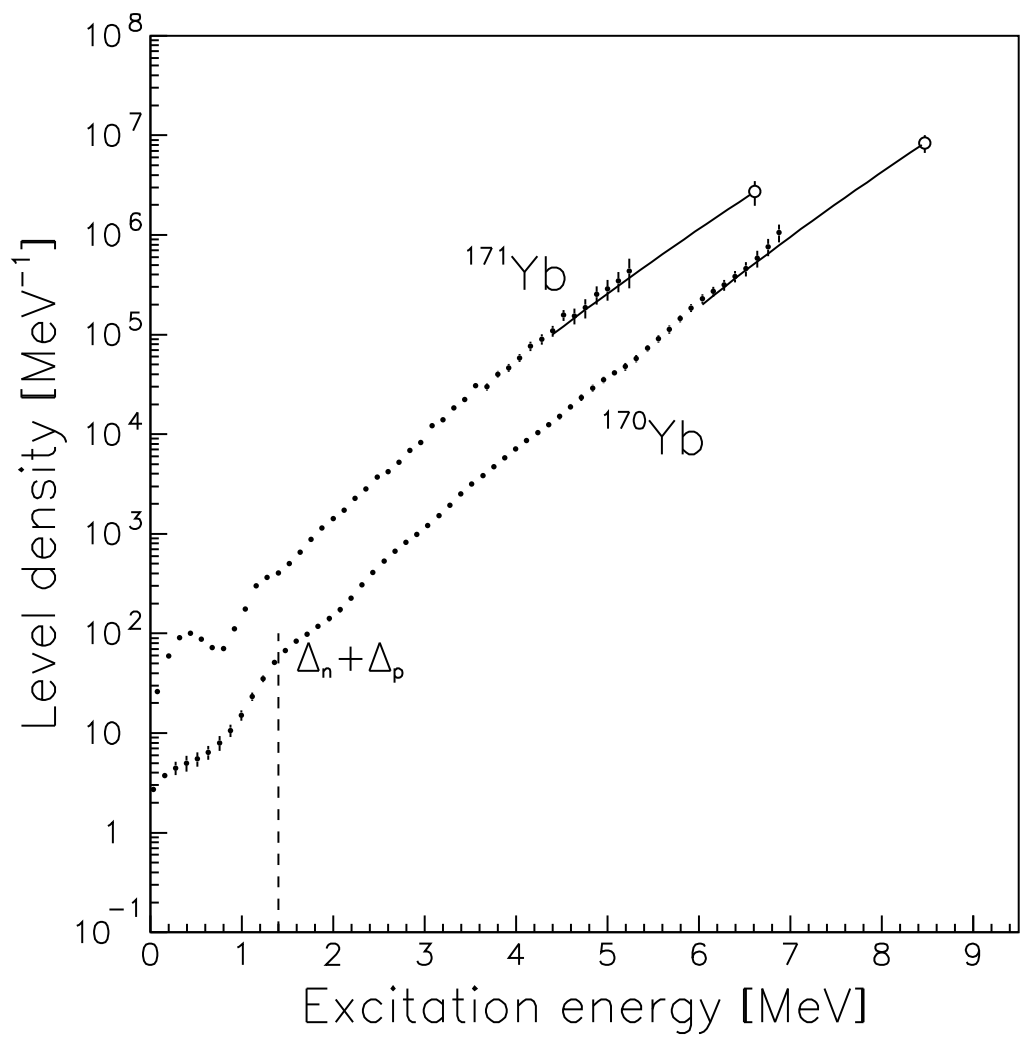

FIGURE 2. The level density of ${ }^{170} \mathrm{Yb}$ and ${ }^{171} \mathrm{Yb}$.

In Figure 3 the ratio of the exponential level density and the experimental level density is plotted as a function of excitation energy $E$. The ratio is shown between the two energies where the fit at the low- and high-energy ends stopped. Fluctuations around the straight line indicates deviation of the observed level density from the exponential level density. There are structures near excitation energies 2, 3.5, and $5.2 \mathrm{MeV}$ seen as deviations from the relative level density $\rho(U) / \rho_{F e r m i}(U)=1$. However, the magnitude of these deviations is within two $\sigma$. 


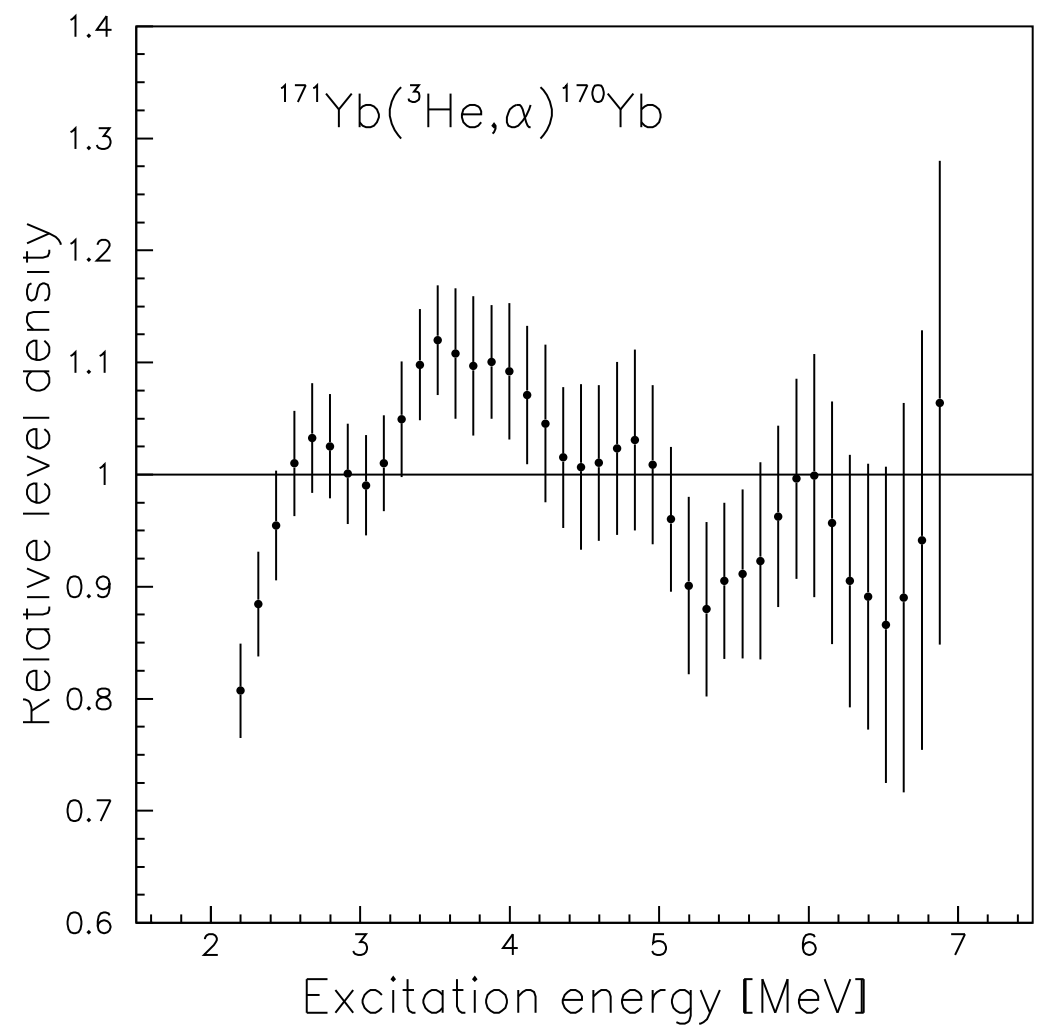

FIGURE 3. The ratio of the level density predicted by the Fermi gas model for ${ }^{170} \mathrm{Yb}$ and the experimental level density.

The normalized experimental radiative strength functions for ${ }^{170} \mathrm{Yb}$ and ${ }^{171} \mathrm{Yb}$ are shown in Figs. 4 and 5 . The experimental radiative strength functions are compared to model calculations. In Figs. 4 and 5 the dashed lines show the tail of the Lorentzian GEDR given by Eq. (3). The dash-dotted lines show a sum of the KMF E1 strength (4) and the Weisskopf $M 1$ estimate. The Weisskopf strength overestimates at low $E_{\gamma}$ because the ratio $f_{M 1} / f_{E 1}$ is given at $E_{\gamma} \approx 7 \mathrm{MeV}$, thus not applicable at low $E_{\gamma}$. The dotted lines represent a sum of the KMF $E 1$ strength (4) and the Lorentzian $M 1$ given by Eq. (6).

The solid lines represent a fit to the data with

$$
f=K\left(f_{E 1}+f_{M 1}\right)+f_{\mathrm{py}}
$$

where $f_{E 1}$ and $f_{M 1}$ are given by Eqs. (4) and (6). Parameters are taken from reference [13]. The pygmy resonance $f_{\mathrm{py}}$ is added to the strength in ${ }^{171} \mathrm{Yb}$ where there is a clear anomaly in the data.

The ratio of the experimental strength function and the fit to $E_{\gamma}^{n}$ provides a qualitative view of the fine structure in the strength function. In Fig. 6 the $\gamma$-ray strength function ratio is plotted as a function of $\gamma$-ray energy. A systematic deviation from the straight line near $E_{\gamma} \sim 3.4 \mathrm{MeV}$ indicates that there is a noticable bump in the strength function. Quantitatively, the parameters for the pygmy resonance are obtained by including a Lorentzian $f_{\text {py }}$ to the fit function (8). The parameters are listed in Table 1 along with results obtained from the ${ }^{172} \mathrm{Yb}\left({ }^{3} \mathrm{He}, \alpha \gamma\right){ }^{171} \mathrm{Yb}$ experiment [11]. The energy and width of the pygmy resonance in ${ }^{171} \mathrm{Yb}$ from the two experiments agree well. The cross section for the pygmy resonance from the ${ }^{172} \mathrm{Yb}\left({ }^{3} \mathrm{He}, \alpha \gamma\right){ }^{171} \mathrm{Yb}$ experiment is somewhat larger.

A similar resonance structure was observed in most of the rare-earth nuclei investigated by the Oslo method. However, for ${ }^{170} \mathrm{Yb}$ the strength function has larger fluctuations. Therefore it is difficult to conclude from present data whether there is a pygmy resonance for ${ }^{170} \mathrm{Yb}$. Thus $f_{\mathrm{py}}$ in Eq. (8) is taken to be zero for ${ }^{170} \mathrm{Yb}$. The shape of the strength function suggests that the pygmy resonance in ${ }^{170} \mathrm{Yb}$ may not be described by a Lorentzian but could be split. More experimental evidence is necessary to clarify this issue. Since $f_{\text {py }}$ in Eq. (8) accounts for the resonant 


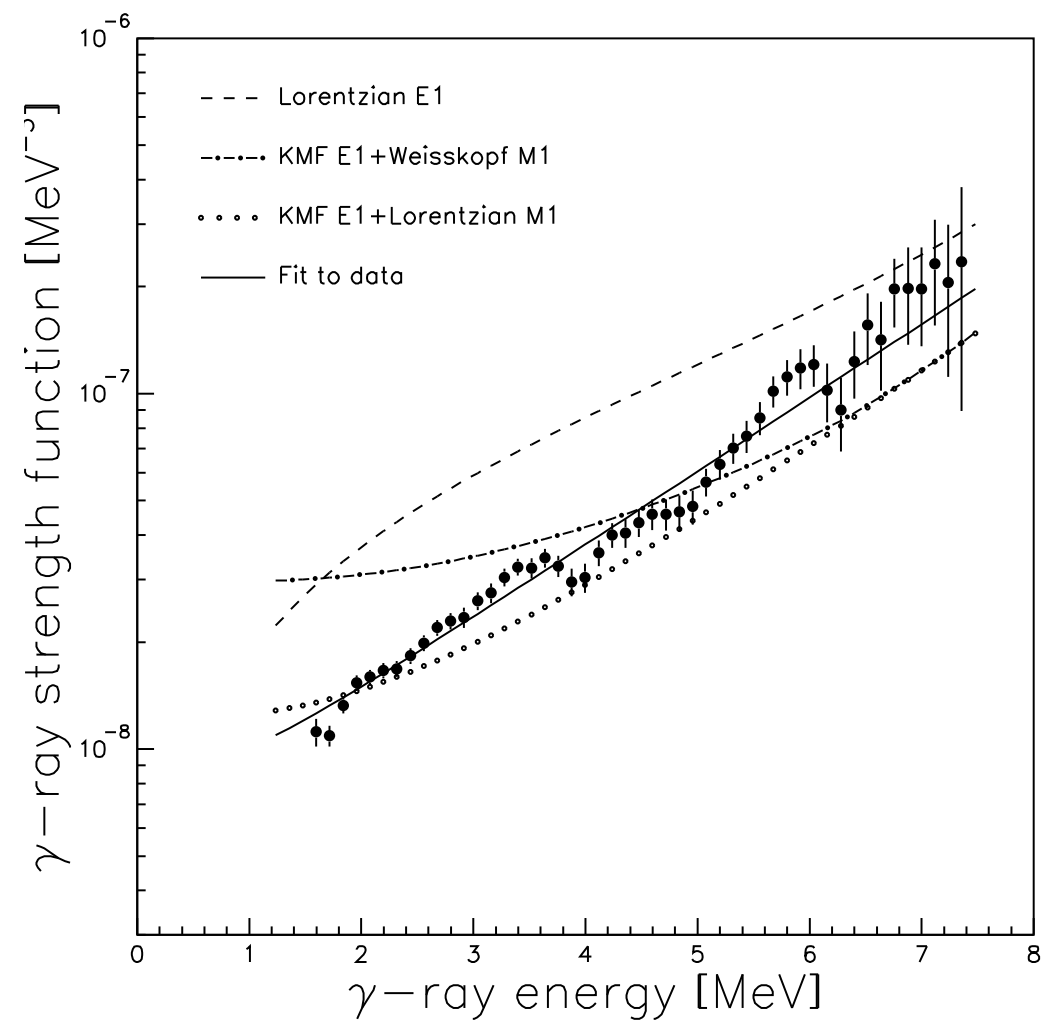

FIGURE 4. The radiative strength function of ${ }^{170} \mathrm{Yb}$. The experimental data is fitted (solid line) using a function given by Eq. (8) with $f_{\text {py }}=0$ and compared to several models.

TABLE 1. The parameters for the pygmy resonance in ${ }^{171} \mathrm{Yb}$ obtained from a previous experiment [11] and from the present work

\begin{tabular}{llllll}
\hline Experiment & $\begin{array}{l}E_{\mathrm{py}} \\
\mathrm{MeV}\end{array}$ & $\begin{array}{l}\sigma_{\mathrm{py}} \\
\mathrm{mb}\end{array}$ & $\begin{array}{l}\Gamma_{\mathrm{py}} \\
\mathrm{MeV}\end{array}$ & $\begin{array}{l}T \\
\mathrm{MeV}\end{array}$ & $K$ \\
\hline Reference [11] & $3.35(6)$ & $0.65(7)$ & $0.97(16)$ & $0.34(3)$ & $1.22(10)$ \\
Present & $3.53(10)$ & $0.35(6)$ & $1.04(18)$ & $0.31(3)$ & $1.01(4)$ \\
\hline
\end{tabular}

structure, the overall normalization factor $K$ should be equal to 1 . For ${ }^{171} \mathrm{Yb}$ where the pygmy resonance was fit using a non-zero $f_{\text {py }}$, we obtained $K=1.01 \pm 0.04$. However for ${ }^{170}$ Yb where $f_{\text {py }}$ was taken to be zero, the overall factor $K$ was $1.20 \pm 0.09$. The fact that it deviates from 1 is indicative of an additional strength in the unresolved background (i.e. pygmy resonance). The temperature $T$ in Eq. (4) is also treated as a free parameter. A fit to the experimental data yields $T=0.42 \pm 0.04 \mathrm{MeV}$ for ${ }^{170} \mathrm{Yb}$ and $T=0.31 \pm 0.03 \mathrm{MeV}$ for ${ }^{171} \mathrm{Yb}$.

\section{SUMMARY}

The level densities and radiative strength functions in ${ }^{170} \mathrm{Yb}$ and ${ }^{171} \mathrm{Yb}$ are obtained from the ${ }^{171} \mathrm{Yb}+{ }^{3} \mathrm{He}$ experiment. The observed level densities provide new values for excitation energies above $\sim 2 \mathrm{MeV}$ where the tabulated levels are incomplete. Because of the large fluctuations in the radiative strength of ${ }^{170} \mathrm{Yb}$, a pygmy resonance in ${ }^{170} \mathrm{Yb}$ is not readily apparent. The radiative strength function in ${ }^{171} \mathrm{Yb}$ exhibits a resonance structure (pygmy resonance) similar to 


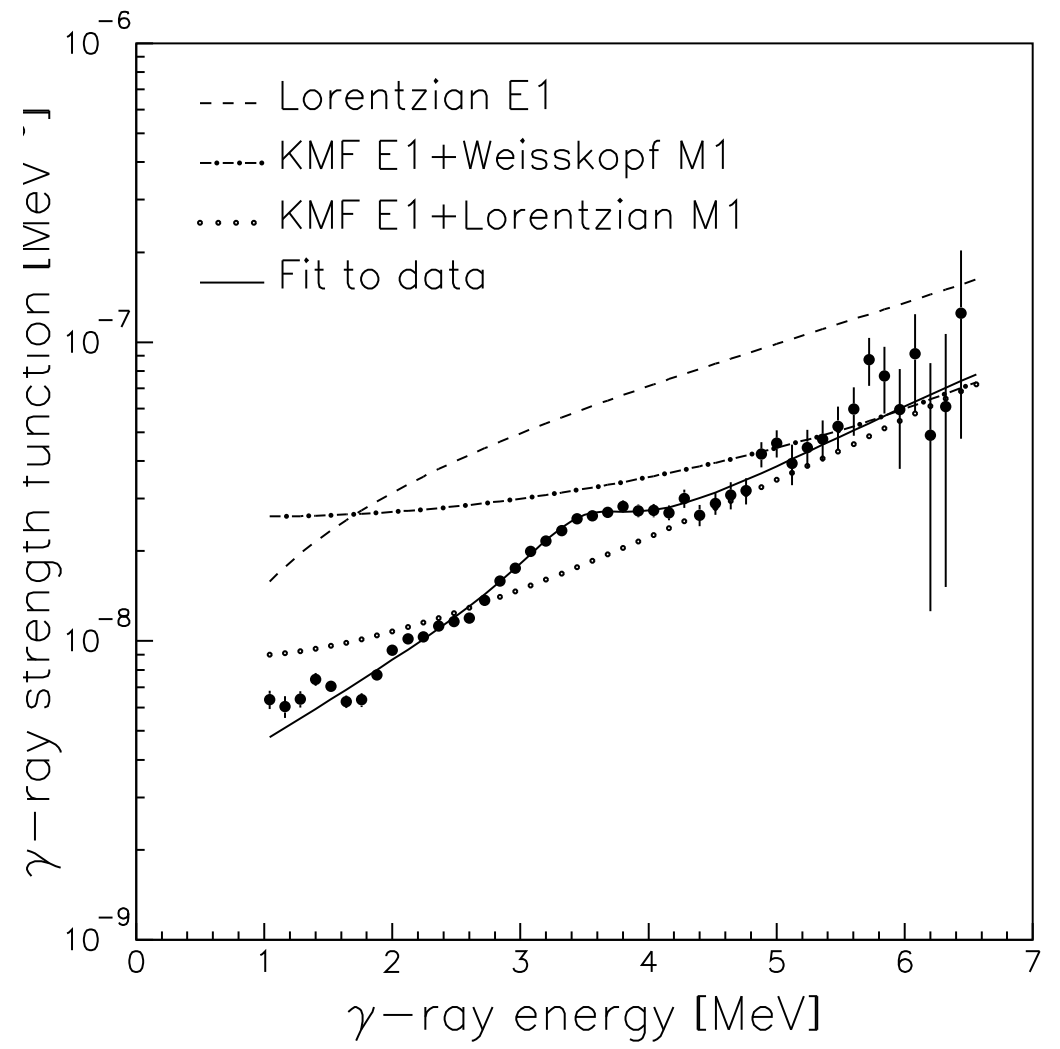

FIGURE 5. The radiative strength function of ${ }^{171} \mathrm{Yb}$. The experimental data is fitted (solid line) using a function given by Eq. (8) and compared to several models. The pygmy resonance parameters are determined from the fit.

that observed in previously measured nuclei. The parameters for this pygmy resonance were obtained and compared to values from the ${ }^{172} \mathrm{Yb}\left({ }^{3} \mathrm{He}, \alpha\right)^{171} \mathrm{Yb}$ reaction. There is good agreement between the two measurements.

\section{ACKNOWLEDGMENTS}

This work was supported in part by the U.S. Department of Energy Grants No. DE-FG03-03NA00076 and No. DEFG02-97-ER41042. Part of this work was performed under the auspices of the U.S. Department of Energy by the University of California, Lawrence Livermore National Laboratory under contract No. W-7405-Eng-48. Financial support from the Norwegian Research Council (NFR) is gratefully acknowledged.

\section{REFERENCES}

1. A. Gilbert and A.G.W. Cameron, Can. J. Phys. 43, 1446 (1965).

2. T. von Egidy, H. H. Schmidt, and A.N. Behkami, Nucl. Phys. A481, 189 (1988).

3. S.S. Dietrich and B.L. Berman, At. Data Nucl. Data Tables 38, 199 (1988).

4. J. Kopecky and M. Uhl, Phys. Rev. C 41, 1941 (1990).

5. A. Schiller, L. Bergholt, M. Guttormsen, E. Melby, J. Rekstad, and S. Siem, Nucl. Instrum. Methods Phys. Res. A 447, 498 (2000).

6. M. Guttormsen, T. S. Tveter, L. Bergholt, F. Ingebretsen, and J. Rekstad, Nucl. Instrum. Methods Phys. Res. A 374, 371 (1996).

7. M. Guttormsen, T. Ramsøy, and J. Rekstad, Nucl. Instrum. Methods Phys. Res. A 255, 518 (1987). 


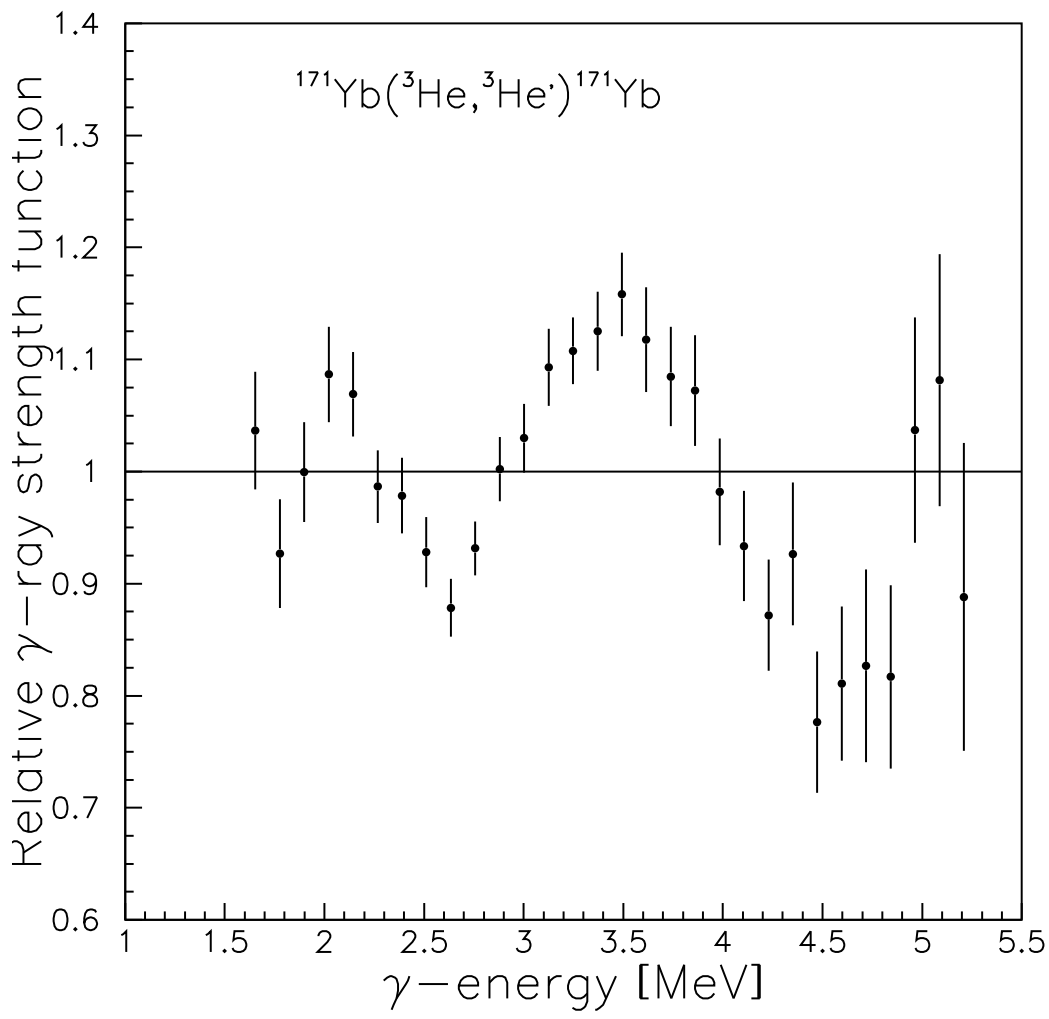

FIGURE 6. The relative radiative strength function of ${ }^{171} \mathrm{Yb}$. The ratio of the observed radiative strength and the smooth function obtained from fitting Eq. (8) to data is shown as a function of $\gamma$-ray energy.

8. D.M. Brink, Ph.D. thesis, Oxford University, 1955.

9. P. Axel, Phys. Rev. 126, 671 (1962).

10. R.B. Firestone and V.S. Shirley, Table of Isotopes, 8th ed., Wiley, New-York, 1996, Vol. II.

11. A. Voinov, M. Guttormsen, E. Melby, J. Rekstad, A. Schiller, and S. Siem, Phys. Rev. C 63, 044309 (2001).

12. S.G. Kadmensky, V.P. Markushev, and V.I. Furman, Yad. Fiz. 37, 277 (1983), [Sov. J. Nucl. Phys. 37, 165 (1983)].

13. Handbook for Calculations of Nuclear Reactions Data, IAEA, Vienna, Report No. IAEA-TECDOC-1024, 1998.

14. S. Siem, M. Guttormsen, K. Ingeberg, E. Melby, J. Rekstad, A. Schiller, and A. Voinov, Phys. Rev. C 65, 044318 (2002).

15. J. Dobaczewski, P. Magierski, W. Nazarewicz, W. Satuła, and Z. Szymański, Phys. Rev. C 63, 024308 (2001).

16. E. Melby, L. Bergholt, M. Guttormsen, M. Hjorth-Jensen, F. Ingebretsen, S. Messelt, J. Rekstad, A. Schiller, S. Siem, and S.W. Ødegård, Phys. Rev. Lett., 83, 3150 (1999).

17. E. Tavukcu, J. Becker, L.A. Bernstein, P.E. Garrett, M. Guttormsen, G.E. Mitchell, J. Rekstad, A. Schiller, S. Siem, A. Voinov, and W. Younes, AIP Conf. Proc., 656, 136 (2002).

18. D.J. Dean and M. Hjorth-Jensen, Rev. Mod. Phys., 75, 607 (2003). 